\begin{tabular}{|c|c|c|c|c|c|c|c|c|}
\hline \multicolumn{2}{|c|}{ I 894} & \multicolumn{3}{|c|}{ Eastern Time } & $p$ & \multicolumn{2}{|c|}{ Eastern Time } & $s$ \\
\hline April & \multirow[t]{2}{*}{2} & \multicolumn{3}{|c|}{$15^{\mathrm{h}} 22^{\mathrm{m}_{1}} 6^{\mathrm{s}}$} & $86: 63$ & \multicolumn{2}{|c|}{$15^{\mathrm{h}} 27^{\mathrm{m}} \mathrm{II}^{\mathrm{s}}$} & I $18 ! 28$ \\
\hline & & I 5 & 37 & 3 & $87 \cdot 31$ & I 53 I & $4 \mathrm{I}$ & I I $7 \cdot 56$ \\
\hline \multirow{4}{*}{\multicolumn{2}{|c|}{15}} & 12 & 22 & 25 & 78.80 & 1225 & 59 & 39.64 \\
\hline & & 12 & 34 & 5 & 78.49 & 1228 & 58 & $39.6 \mathrm{I}$ \\
\hline & & & 50 & 37 & 75.68 & 1453 & $4^{6}$ & $35 \cdot 17$ \\
\hline & & 14 & 58 & 44 & 76.06 & I 456 & 33 & 34.88 \\
\hline \multirow{2}{*}{\multicolumn{2}{|c|}{24}} & 14 & 28 & 48 & 80.36 & 1439 & 42 & 98.87 \\
\hline & & 14 & 49 & $5^{2}$ & 80.69 & I 4 I & 3.9 & 98.80 \\
\hline \multirow{2}{*}{\multicolumn{2}{|c|}{25}} & 13 & 22 & 45 & $91 \cdot 30$ & I 328 & 51 & 49.60 \\
\hline & & r 3 & 35 & I & 92.20 & I 331 & 27 & 49.75 \\
\hline \multirow{2}{*}{\multicolumn{2}{|c|}{27}} & I 2 & 8 & I 9 & $267 \cdot 3^{8}$ & 12 I I & $5^{8}$ & $49 \cdot 52$ \\
\hline & & 12 & 17 & 20 & $267 \cdot 52$ & 1214 & 45 & $49 \cdot 34$ \\
\hline & \multirow[t]{4}{*}{9} & 8 & 37 & 50 & 83.16 & $84 \mathrm{I}$ & 19 & $42: 46$ \\
\hline & & 8 & 45 & 27 & 82.96 & 843 & 5 & 42.53 \\
\hline & & 9 & 46 & $5^{6}$ & 82.15 & 950 & 28 & 42.07 \\
\hline & & 9 & 54 & 7 & 82.11 & 952 & 6 & 41.87 \\
\hline \multirow[t]{8}{*}{ May } & \multirow[t]{2}{*}{ I } & I I & 54 & 51 & 252.85 & I I 57 & 50 & 33.46 \\
\hline & & 12 & 4 & 5 & 252.45 & 120 & 42 & $33 \cdot 58$ \\
\hline & \multirow[t]{2}{*}{3} & I 2 & I 3 & 7 & 48.24 & I 2 I 5 & 29 & 17.97 \\
\hline & & 12 & 19 & 44 & $47.9^{8}$ & 1217 & $3^{8}$ & 17.98 \\
\hline & \multirow[t]{2}{*}{9} & 13 & I 2 & $3^{8}$ & $99 \cdot 3^{\circ}$ & I3 16 & 14 & 56.28 \\
\hline & & & 20 & $5^{8}$ & 99.45 & 1317 & 35 & $56.3^{2}$ \\
\hline & \multirow[t]{2}{*}{12} & Io & 20 & 37 & 75.68 & I0 23 & 33 & 77.82 \\
\hline & & 10 & 29 & 27 & 75.78 & 1026 & 27 & 77.96 \\
\hline
\end{tabular}

University of Virginia 1897 March.

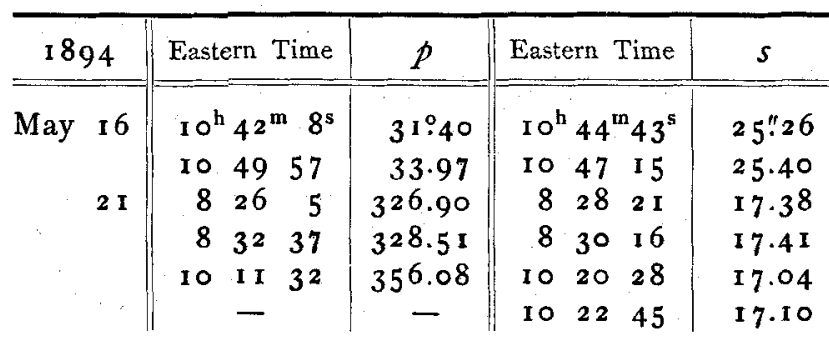

Enceladus-Titan.

\begin{tabular}{|c|c|c|c|c|c|c|c|c|c|}
\hline Lar. & 8 & I 3 & 20 & 9 & 0.70 & & - & & - \\
\hline & 14 & I 6 & $4 \circ$ & 49 & I 12.24 & & - & & - \\
\hline & 23 & $\mp 5$ & 27 & 57 & 307.95 & & - & & - \\
\hline 4 pril & 2 & 15 & 39 & 27 & 252.93 & I 5 & 43 & 49 & 102.85 \\
\hline & & 15 & 51 & 26 & 252.70 & I 5 & 47 & 7 & 103.40 \\
\hline & I 5 & 12 & 41 & 8 & 123.69 & I 2 & 44 & 54 & 76.09 \\
\hline & & 12 & $5 \mathrm{I}$ & 37 & 124.27 & 12 & 48 & 40 & 76.16 \\
\hline & 24 & I 3 & 14 & 45 & I I. IO & 13 & I 8 & 47 & 44.05 \\
\hline & & I 3 & 24 & 30 & I 1.69 & I 3 & 21 & 36 & 44.02 \\
\hline & 25 & I 3 & 37 & 39 & $35 \cdot 5^{6}$ & 13 & 41 & 31 & 56.59 \\
\hline & & I 3 & 47 & 55 & $3^{6.11}$ & I 3 & 44 & 25 & $5^{6.80}$ \\
\hline ay & 9 & I 3 & 36 & I 4 & 301.40 & I 3 & 39 & 42 & 72.06 \\
\hline & & I 3 & 46 & 16 & 301.96 & 13 & 43 & 50 & 72.10 \\
\hline & 10 & I 3 & 5 & 16 & 309.37 & 13 & 7 & $3 I$ & 62.99 \\
\hline & & I 3 & I 2 & 37 & 309.96 & I 3 & 9 & 29 & 62.88 \\
\hline
\end{tabular}

E. O. Lovett.

\title{
New Double Stars and Ring Nebula.
}

The following double stars and ring nebula were discovered by me during 1894 by means of an $8 \frac{1}{2}$ inch reflecting telescope by With and are believed to be new. The double stars have not yet been published, with the exception of Nos. 2 and 4, which are included in a list of suspected new double stars published by R. T. A. Innes in the Monthly Notices of the Royal Astronomical Society, Vol. LV, No. 5 .

All the angles and distances are estimated only. The positions are for 1900 .

No. I. Lacaille 342 .

$$
\text { RA. }=1^{\mathrm{h}} 3^{\mathrm{m}_{5}} \quad \text { Decl. }=-83^{\circ} 47^{\prime} \text {. }
$$

r $894.9 \quad 0^{\circ} \pm \quad 0.8 \pm \quad 8$ and 9

No. 2. Lacaille 1430 .

RA. $=4^{\mathrm{h}}{ }^{\mathrm{m}}{ }^{\mathrm{m}} \cdot 8$ Decl. $=-6 \mathrm{I}^{\circ}{ }^{12}$.

$1894.7 \quad 295^{\circ} \pm \quad$ o."8 6 and 7

Newcastle, New South Wales, I897 March I3.
No. 3. $\varepsilon$ Lupi.

$$
\begin{aligned}
& \text { RA. }=I 5^{\mathrm{h}} 15^{\mathrm{m}} \cdot 9 \quad \text { Decl. }=-44^{\circ} 20^{\circ} \text {. } \\
& \text { I894.9 } 305^{\circ} \pm \quad 0.8 \quad 3.5 \text { and } 5
\end{aligned}
$$

No. 4. $\xi$ Pavonis.

RA. $=18^{\mathrm{h}} 14^{\mathrm{m}}$ Decl. $=-6 \mathrm{I}^{\circ} 32^{\prime}$.

$1894.7 \quad 140^{\circ} \pm \quad 4^{\prime \prime} \pm \quad 5$ and I I

$$
\text { No. 5. Cord. GC. } 26287 \text {. }
$$

RA. $=19^{\mathrm{h}} 7^{\mathrm{m}}{ }_{1} \quad$ Decl. $=-66^{\circ} 50^{\circ}$.

$$
\text { I } 894.8 \quad 40^{\circ} \pm \quad 0.5 \pm \quad 6 \text { and } 8
$$

The ring nebula found on 1894 , June 4 , and published in the Journal of the British Astronomical Association, Vol. VI, No. 5, has been observed by Mr. Baracchi, Director of the Melbourne Observatory, by means of the Great Reflector of 4 feet aperture. To him it appears almost circular and $1: 5 \pm$ in diameter, the central portion being not quite devoid of nebulous matter.

Its position is RA. $2 \mathrm{I}^{\mathrm{h}} 53^{\mathrm{m}} 10^{\mathrm{s}}$, Decl. $-39^{\circ} 53^{\prime} 42^{\prime \prime}$.

Walter F. Gale.

Planet (130) Elektra. Correction der Ephemeride im Berl. Jahrb. für r899: r897 Mai $29 \Delta \alpha=+15^{5} \cdot 35$, $\Delta \delta=-59.5$. 